Abstract AWE-06 Table 1

\begin{tabular}{|c|c|c|c|c|}
\hline & & Monotherapy $(n=51)$ & Combination therapy $(n=17)$ & Rescue Therapy $(n=7)$ \\
\hline \multirow[t]{2}{*}{ Blatchford } & Median & 10 & 9 & 11 \\
\hline & IQR & $7-12$ & $7-14$ & $10-13$ \\
\hline \multirow[t]{2}{*}{ Rockall } & Median & 8 & 8 & 7 \\
\hline & $\mathrm{IQR}$ & $7-9$ & $7-9$ & $4-7$ \\
\hline \multicolumn{2}{|c|}{ Haemostasis achieved } & $51 / 51(100 \%)$ & $15 / 17(88 \%)$ & $7 / 7(100 \%)$ \\
\hline \multicolumn{5}{|c|}{ Rockall 7 and 8: Predicted rebleed rate: $25-40 \%$} \\
\hline \multicolumn{2}{|l|}{ Re-bleed } & $4 / 44(9 \%)$ & $2 / 14(14 \%)$ & $1 / 7(14 \%)$ \\
\hline \multicolumn{5}{|c|}{ Rockall 7 predicted mortality rate: $20-30 \%$} \\
\hline \multicolumn{5}{|c|}{ Rockall 8 predicted mortality rate: $40-45 \%$} \\
\hline \multicolumn{2}{|c|}{ All cause 7-day mortality } & $2 / 44(5 \%)$ & $0 / 14$ & $0 / 7$ \\
\hline \multicolumn{2}{|c|}{ All cause 30-day mortality } & $9 / 44(21 \%)$ & $3 / 14(21 \%)$ & $0 / 7$ \\
\hline
\end{tabular}

secondary to tumours as a monotherapy, dual-therapy with standard haemostatic techniques or rescue therapy. Haemostasis was defined as the cessation of bleeding within 5 minutes of the application of hemospray.

Results 75 patients with tumoural UGIBs were recruited (51 males, 24 females, 22/75 (29\%) oesophageal, 49/75 (65\%) gastric, $4 / 75$ (5\%) doudenal). The median rockall score was 8 (IQR,7-9).

Immediate haemostasis was achieved in $73 / 75$ (97\%) of patients, 7/65 (11\%) had a rebleed, 12/65 (18\%) died within 30 days (all-cause mortality). Based on the baseline average total rockall score, the expected rebleed rate is $25-40 \%$, and expected mortality rate was $40-45 \%$ in our cohort.

$100 \%$ immediate haemostasis was achieved in duodenal and gastric tumours, and $91 \%$ in oesophageal tumours.

Outcomes with mono/combination therapy and rescue therapy (table1)

Conclusions Hemospray is effective for achieving immediate haemostasis in UGIBs secondary to upper GI tumours, which are considered difficult to treat.

When considering average rockall score the rebleed and mortality rate is better than predicted rates. Haemostasis is achieved in the majority allowing for patient stabilization and providing time for surgery/radiotherapy.

\section{AWE-07 APOLLO OVERSTITCH DEVICE FOR ENDOSCOPIC REVISION OF ROUX-EN-Y GASTRIC-BYPASS, UPDATES FROM THE LARGEST UK SERIES}

Ahmed Ghanem, Vasha Kaur*, Marta Mano Lopes, Jonathan Cousins, Ahmed Ahmed, Dav Bansi. St. Mary's Hosptal, Imperial College Healthcare NHS trust, London, UK

\subsection{6/gutjnl-2019-BSGAbstracts.25}

Introduction The Apollo OverStitch is a minimally invasive endoscopic suturing device which allows full thickness suturing without the need for surgery

Roux-en-Y gastric bypass (RYGB) can achieve up to $60 \%$ weight loss 2 years after surgery but $30 \%$ of patients will regain their weight within 2 years. The options for this group of patients are limited; redo surgery can be challenging with a greater risk of complications. Endoscopic revision of the gastro-jejunal anastomosis using the Apollo OverStitch device now offers an alternative option in these patients to achieve further weight loss. Here we report our experience using this device, which is the largest patient cohort in the UK to date.

Method Between April 2017 and December 2018, we have used the Apollo OverStitch device in 23 patients who had regained weight after an initial RVGB. All patients were discussed initially at our bariatric MDT. All patients underwent a prior gastroscopy to ensure a stoma size of at least $2 \mathrm{~cm}$. All cases were done under general anaesthetic.

Results Here we report the follow up data for our cohort. 91\% of patients were females. Mean weight loss at early follow up (mean 82 days) was $6.7 \%$ and at late follow up (mean 342 days) was 9.9\%. One patient had a re-do procedure, having dropped from $104 \mathrm{~kg}$ to $95 \mathrm{~kg}$ and then further to $88.8 \mathrm{~kg}$. There were no procedure related complications.

Conclusion Endoscopic revision of the RYGB stoma using the Apollo OverStitch device is an effective method of achieving further weight loss in these patients. The effects are sustained at 1 year. We are now developing our technique to incorporate more bites for each suture placed, as well as following the first line of sutures with a second in order to achieve a tighter effect on reducing the stoma size. We have done a further 7 patients since, making our cohort the largest in the UK. We propose that this management option be considered in all patients with weight regain after RYGB and a gastrojejunal stoma $>2 \mathrm{~cm}$ at gastroscopy.

\section{Posters}

\section{PTH-001 SEMS IS A RISK FACTOR FOR POST-ERCP PANCREATITIS: EXPERIENCE FROM A HIGH-VOLUME UK HOSPITAL} 1,2Alaa Abdelwareth*, 'Ravi Madhotra. ${ }^{1}$ Milton Keynes University Hospital, Milton Keynes,
UK; ${ }^{2}$ Assiut University Hospital, Egypt

\subsection{6/gutjnl-2019-BSGAbstracts.26}

Introduction Studies have attempted to examine the risk of post-ERCP pancreatitis (PEP) with the placement of biliary self-exapandable metal stent (SEMS) with conflicting results. The aim of this study is to investigate whether biliary SEMS is a potential risk factor for PEP.

Methods All patients who underwent ERCP between January 2016 and May 2017 at Gastrointestinal Endoscopy Unit at 
Milton Keynes University Hospital (MKUH) were included in this retrospective study. Patients included in this study underwent first time ERCP as indicated, including patients who developed PEP. PEP was defined according to consensus criteria. Patients who developed complications other than PEP, those who had failed procedure due to anatomical deformity, previous ERCP, or active pancreatitis at the time of ERCP were excluded.

Results A total of 260 patients who underwent ERCP between January 2016 and May 2017 were included in this retrospective study. After applying exclusion criteria 29 patients were excluded and 231 patients were included in the final analysis. PEP occurred in $8.6 \%(20 / 231)$ of all patients. Normal serum bilirubin was the only patient related risk factor which showed statistically significant association with PEP on univariate analysis $(\mathrm{P}=0.001)$. On univariate analysis of procedure risk factors we found that pancreatic guidewire cannulation, pancreatic contrast injection, and biliary metal stent insertion showed statistically significant association with PEP. Multivariate analysis after binary logistic regression analysis revealed that normal serum bilirubin $(\mathrm{P}<0.001)$ and bilary metal stent insertion $(\mathrm{P}=0.001)$ are independent risk factors for PEP. Pancreatic guidewire cannulation and pancreatic contrast injection increased the risk of PEP, but this was not statistically significant on the multivariate analysis.

Conclusions Normal serum bilirubin and biliary metal stent placement were independent risk factors for the occurrence of PEP. Further research is needed to investigate the etiological factors contributing to the development of PEP after biliary SEMS insertion. This will help to develop strategies to reduce SEMS related pancreatitis.

\section{PTH-002 OUT OF HOUR COLONOSCOPY; IS THE QUALITY AS GOOD AS IN HOUR COLONOSCOPY?}

Shimaa Afify*, Thomas Archer, Mo Thoufeeq. Sheffield Teaching Hospitals, UK

\subsection{6/gutjnl-2019-BSGAbstracts.27}

Introduction Colonoscopy is the 'gold standard' investigation for assessment of the large bowel which detects and prevents colorectal cancer, as well as non-neoplastic conditions. The quality of UK colonoscopy has improved as in 2013 The Joint Advisory Group (JAG) on GI Endoscopy suggests monitoring key performance indicators such as cecal intubation rate (CIR) and the adenoma detection rate (ADR). There has been a drive to improve utilization of endoscopy units nationwide by offering out of hours $(\mathrm{OOH})$ endoscopy to deal with increasing service demands. Our aim was to investigate the quality of colonoscopies carried out during evening and Saturday lists in our unit and compare against BSG standards of quality for colonoscopies. The endoscopy team consisted of our team.

Method We retrospectively collected and analyzed demographical and procedure related data for non- screening colonoscopies performed between January 2016 and April 2018. Procedures were grouped according to the day of the week and timing of session (weekday, evening and Saturday). We also compared those performed by advanced colonoscopist and non-advanced colonoscopist. Advanced colonoscopist was defined as a BCSP colonoscopist or an advanced EMR colonscopists.

Results There were a total of 13642 colonoscopies that were carried out. $26.3 \%(n=3601)$ involved patients were $>70$ years and $73.7 \%(n=10041)$ were $<70$ years. Table 1 summarizes the differences between weekdays, evening and Saturdays' colonoscopies regarding the CIR and ADR. We noted that the KPIs (ADR, CIR) met the JAG standards. Advanced colonoscopists had better KPIs when compared to the non-advanced colonoscopists.

Conclusion JAG standards were maintained during colonoscopies done on weekdays, evening and Saturdays. Advanced colonoscopists had higher CIR and ADRs.

\begin{tabular}{|c|c|c|c|c|c|c|c|c|}
\hline & & \multicolumn{2}{|c|}{ Weekdays } & \multicolumn{2}{|c|}{ Evenings } & \multicolumn{3}{|c|}{ Saturdays } \\
\hline & & No. & $\begin{array}{l}\text { Rate } \\
(\%)\end{array}$ & No. & $\begin{array}{l}\text { Rate } \\
(\%)\end{array}$ & No. & $\begin{array}{l}\text { Rate } \\
(\%)\end{array}$ & $\begin{array}{l}\mathrm{p} \\
\text { value }\end{array}$ \\
\hline \multirow[t]{2}{*}{ Total } & ADR & 10775 & 28.5 & 1397 & 30.2 & 1469 & 24.6 & $0.002^{\mathrm{a}}$ \\
\hline & CIR & & 90.4 & & 90.6 & & 91.4 & 0.495 \\
\hline Advanced & ADR & 954 & 42.6 & 136 & 32.4 & 197 & 34.0 & $0.012^{b}$ \\
\hline colonoscopist & CIR & & 93.7 & & 96.3 & & 94.9 & 0.423 \\
\hline Non-advanced & ADR & 9821 & 27.1 & 1261 & 23.5 & 1272 & 23.1 & $0.001^{c}$ \\
\hline colonoscopist & CIR & & 90.1 & & 90.0 & & 90.8 & 0.704 \\
\hline advanced vs. & $p$ value (ADR) & $<0.001$ & & 0.022 & & 0.001 & & \\
\hline $\begin{array}{l}\text { Non-advanced } \\
\text { colonoscopist }\end{array}$ & $p$ value (CIR) & $<0.001$ & & 0.016 & & 0.055 & & \\
\hline
\end{tabular}

CIR: Caecal intubation rate, ADR: adenoma detection rate, a: Saturday is statistically significant than other days in ADR, b: all are statistically significant, c: week days are statistically significant from evenings and Saturdays

\section{PTH-003 DOES SIZE MATTER: HOW ACCURATE ARE OUR POLYP- SIZING METHODS?}

${ }^{1}$ Lulia Al-Hillawi ${ }^{*}$ 'Sun Mi Ha, ${ }^{2}$ Amir Saifuddin, ${ }^{2}$ Debra Uttridge, ${ }^{2}$ Chirag Kothari, ${ }^{1}$ Bijay Baburajan. 'Maidstone And Tunbridge Wells NHS Trust, Maidstone, UK; ${ }^{2}$ Dartford and Gravesham NHS Trust, Dartford, UK

\subsection{6/gutjnl-2019-BSGAbstracts.28}

Introduction Correct sizing of a colonic polyp at the time of lower gastrointestinal (LGI) endoscopy is key in dictating polypectomy method, use of tattoo, and future surveillance in colorectal cancer prevention (CRC). There are no accepted standards for how to size a polyp and therefore it is endoscopist-dependent and subject to inter-observer variation. Visual estimation (VE), or the use of open forceps (OF) to are the two most widely-used methods. There are limited studies on polyp-sizing at LGI endoscopy but they would suggest that the use of a measurement guide such as OF would improve size estimation. Our aim was to ascertain which polyp-sizing technique method is preferred. A secondary aim was to see if those using OF knew the size of the forceps being used.

Methods A 6 question survey was completed by endoscopists and nursing staff from Endoscopy Departments in 3 hospitals in West Kent using an online survey tool. Questions included asking for the respondent to identify how to position the scope and forceps based on photographs, and to identify the correct size of the OF when fully open.

The size of the open forceps of the Boston Scientific $(C)$ Standard $2.8 \mathrm{~mm}$ Radial $\mathrm{Jaw}^{\mathrm{TM}_{4}}$ single-use biopsy forceps was measured against a standard UK ruler.

Results There were a total of 27 respondents: $81 \%$ were endoscopists and 19\% nurse observers. 6 trainee endoscopists completed the survey. 